\title{
Predictors of neurological outcome in the emergency department for elderly patients following out-of- hospital restoration of spontaneous circulation
}

BY KATSUHIRO NAGATA, JUNYA TSURUKIRI, KEIKO UENO, SHIRO MISHIMA

\begin{abstract}
Aims. Survival rates for cardiac arrest in acute medicine are higher following outof-hospital restoration of spontaneous circulation (OH-ROSC). However, data pertaining to OH-ROSC is limited in the elderly population. We aimed to assess the predictors of neurological outcome among elderly patients with OH-ROSC.

Methods. We retrospectively analyzed the data of patients 65 years and older who achieved OH-ROSC and who presented to the emergency department (ED) between 2009 and 2013. The following parameters were considered: age, sex, medical history, vital signs, blood values, initial electrical rhythm, witnessed cardiac arrest, bystander cardiopulmonary resuscitation, resuscitation duration, attempted defibrillation, and neurological outcome. Neurological outcomes were evaluated 3 months after cardiac arrest, using the cerebral performance category (CPC) score, and were classified into two groups: favorable outcome $(\mathrm{CPC}=1-2)$ and unfavorable outcome $(\mathrm{CPC}=3-5)$.
\end{abstract}

Results. Fifty-five patients were studied, of which 21 and 34 patients were classified as having favorable and unfavorable outcomes, respectively. The 
following values were associated with favorable outcomes: resuscitation duration, initial cardiac rhythm, base excess, $\mathrm{pH}$, lactate levels, the motor response on the Glasgow Coma Scale (GCS), and the number of patients with GCS $\leq 8(\mathrm{p}<0.01)$. Logistic regression analysis confirmed that motor response scores and lactate levels were independent predictors of neurological outcomes.

Conclusions. Lactate levels and GCS motor response measured immediately at ED arrival are likely to be useful to assess the neurological outcomes among elderly patients with OH-ROSC.

Key words: age, basic life support, cardiac arrest, prediction, resuscitation

\section{Introduction}

In a nationwide Japanese study on the elderly population, the 1-month survival rate was $6.9 \%$, with only $2.8 \%$ achieving favorable neurological outcomes after sudden out-of-hospital cardiac arrest (OHCA). (1) Out-of-hospital cardiac arrest is associated with a high mortality rate, making it an important public health concern. However, advancements in care after restoration of spontaneous circulation (ROSC) have been demonstrated to increase survival and ameliorate neurological outcomes; this is not only for ventricular fibrillation (VF) but also for pulseless electrical activity (PEA)/asystole. (2) Moreover, the survival rates after post-ROSC care are higher with out-of-hospital ROSC (OH-ROSC) than without OH-ROSC. (3-5)

Although resuscitation efforts should focus on achieving OH-ROSC, little is known about elderly patients that arrive at the emergency department (ED) after achieving OH-ROSC. (6-8) Encountering an increased incidence of OHCA in the elderly, emergency physicians urgently need improved prehospital resuscitation approaches and updated information. This study aimed to assess the predictors of good neurological outcomes among elderly patients with OH-ROSC after sudden cardiac arrest at a tertiary emergency center.

\section{Methods}

\section{Hospital and setting}

A retrospective, observational study was conducted at the ED of Tokyo Medical 
University, Hachioji Medical Center, between April 2009 and March 2013. The study design received the appropriate ethics committee approval.

\section{Patients}

The present study included all patients aged 65 years and older who achieved $\mathrm{OH}-$ ROSC before arrival at the ED. Therapeutic hypothermia was available at the study center. We excluded patients with a Glasgow Coma Scale (GCS) score of 15, trauma, intracranial hemorrhage (i.e. subarachnoid hemorrhage or intracranial hematoma), or terminal diseases. We also excluded patients with ongoing or relapsed cardiac arrest on arrival, despite earlier OH-ROSC.

According to our standard practice, therapeutic hypothermia was applied to comatose patients with GCS $\leq 8$, regardless of the initial rhythms. The application in patients with a risk of bleeding or severe shock was at the discretion of the emergency physicians treating the patient. Hypothermia was initiated using circulating water blankets, with a target temperature of $33 \pm 0.5^{\circ} \mathrm{C}$ maintained for

$24 \mathrm{~h}$. Sedation was induced by intravenous midazolam and fentanyl with dose adjustments as needed. Paralysis was induced with pancuronium to prevent shivering during therapeutic hypothermia. Furthermore, all patients received standard post-resuscitation care. Sedation was stopped after rewarming, except for those patients requiring further intensive care.

\section{Data collection}

The following patient characteristics were retrieved from charts and electrocardiograms: age, sex, medical history, first recorded electrical rhythm by emergency medical service (EMS) or bystander, whether OHCA was witnessed by a bystander, whether a bystander performed cardiopulmonary resuscitation (CPR), resuscitation duration, whether EMS or bystander defibrillation was attempted, and any relevant outcomes. On arrival at the ED, the following were immediately evaluated: GCS (specifically motor response), blood pressure, body temperature, and blood samples. Hematological samples were sent for hemoglobin, hematocrit, potassium, sodium, creatine phosphokinase (CPK), creatinine, base excess (BE), lactate, $\mathrm{pH}$, prothrombin international normalized ratio (PT-INR), D-dimer, lactate dehydrogenase (LD), and albumin levels. All values are readily available in EDs and have previously been reported as predictors of ROSC, survival, or neurological outcomes. (9-15) In the prehosiptal setting, either endotracheal or tracheal intubation was applied to most patients following cardiac arrest. Since the GCS motor response is more accurate than the 
GCS sum score and particularly important for prognosis, we specifically quantified the motor response of GCS. (16)

All patients were examined using the cerebral performance category (CPC) score, 3 months after their OHCA and were classified into either favorable outcome (CPC $=1-2)$ or unfavorable outcome $(\mathrm{CPC}=3-5) \cdot(17)$

\section{Statistical analysis}

Data from all eligible patients were analyzed. SPSS Statistics version 21 (IBM Japan, Tokyo, Japan) was used for statistical analysis. Statistical significance was indicated by $\mathrm{p} \leq \mathrm{0.01}$.

Continuous variables are shown as median values with interquartile ranges. Intergroup differences were statistically assessed using the Mann-Whitney U test for continuous variables and the Fisher exact test for categorical variables. Categorical variables were calculated as ratio (\%) of the frequency of occurrence. Logistic regression analysis was used to model the outcome as a function of collateral score, and covariates were selected using backward selection methodology. Baseline variables potentially associated with an outcome $(\mathrm{P}<0.01)$ were considered for inclusion in the model. The sensitivity, specificity, and positive likelihood ratio at various characteristic cut-off points were calculated, based on analysis of receiver operating characteristic (ROC) curves and the maximum Youden index $(\mathrm{J}=$ sensitivity + specificity -1$)$ determined for each ROC curve.

\section{Results}

Fifty-five patients presenting with OH-ROSC and admitted to our intensive care unit (ICU) were included. Twenty-one patients had favorable neurological outcomes and 34 had unfavorable outcomes. Table 1 compares the baseline characteristics of these patients. Favorable outcomes were significantly associated with the following: lower lactate levels; fewer patients with initial PEA/asystole rhythms, and GCS $\leq 8$; and shorter resuscitation durations. Furthermore, favorable outcomes were significantly associated with the following: initial VF rhythm; higher GCS motor response; and higher base excess, and pH. In logistic regression analysis, GCS motor response [odds ratio (OR), 1.8; 95\% confidence interval (CI), 1.2-2.7; $\mathrm{p}<0.01$ ] and lactate levels [OR, 1.0; 95\% CI, 0.9-1.0; $\mathrm{p}<0.01$ ] were independent predictors of favorable neurological outcomes. 
Figure 1 shows the ROC curves for the predictive factors of neurological outcomes. The areas under the ROC curve (AUC) for a favorable neurological outcome were $0.82(95 \% \mathrm{CI}=0.70-0.94, \mathrm{p}<0.01)$ for lactate level and 0.74 (95\% CI $=0.60-0.88, \mathrm{p}<0.01)$ for motor response on GCS. We therefore used these values to generate sensitivity and specificity values for the lactate cut-off levels shown in table 2. At ED arrival, lactate levels above $70 \mathrm{mg} / \mathrm{dL}$ (7.8 mmol/L) and a GCS motor response $\leq 3$ were predictors of unfavorable neurological outcome.

\section{Discussion}

Our findings offer important information for predicting post-resuscitation outcomes that can support the development of future policies. We demonstrated that key variables differed significantly between elderly patients with favorable and unfavorable outcomes and lactate levels and GCS motor responsiveness measured immediately at ED arrival were associated with favorable outcomes. Analyses of the ROC curves suggested that, at ED, lactate levels of $70 \mathrm{mg} / \mathrm{dL}$ ( 7.8 $\mathrm{mmol} / \mathrm{L}$ ) and a GCS motor response of 3, were the most suitable cut-offs for the prediction of neurological outcomes; none of our elderly patients with levels above these had favorable neurological outcomes.

Serum lactate is well known as a clinical biomarker of tissue hypoperfusion and lactate levels measured at the EDs are associated with high mortality in patients with circulatory shock, trauma and infections. $(18,19)$ They are also associated with mortality and morbidity in elderly with and without infections. (20) Shinozaki et al. indicated that lactate cut-off levels of $12.0 \mathrm{mg} / \mathrm{dL}(1.3 \mathrm{mmol} / \mathrm{L})$ were optimal for predicting favorable outcomes in patients with OHCA $(\mathrm{AUC}=0.74) .(21)$ During cardiac arrest or prehospital postresuscitation phase, there might be microcirculatory dysfunction and metabolic derangements which are associated with ischemia or alteration of pyruvate dehydrogenase activity. The GCS motor response at ED arrival, 24 hours, and 72 hours were the greatest predictive value for determining prognosis after cardiac arrest. $(12,16)$ These findings might help clinicians have greater certainty of a poor prognosis, which can in turn indicate the premature cessation of costly resuscitation attempts in some elderly patients following OH-ROSC. A proper assessment of prognosis also indicates effective counseling for patients and relatives.

Age, body temperature, witness, and/or bystander CPR, successful defibrillation at 
the scene, and coagulation have been reported as predictors of survival among patients with OHCA. $(3,6,9,10,12-14)$ Base excess measured after ROSC at the ED was not found to be an independent predictor for favorable outcomes. (15) In the elderly population, CPR performed by a nonfamily bystander or shockable rhythm were reasonable predictors of outcomes following OHCA. $(1,22)$ Although most published studies have targeted OHCA patients arriving at the ED without prior ROSC, our study demonstrated that the preceding variables, excluding the GCS scale, are not predictors of neurological outcome among elderly patients with OH-ROSC. The emergency medical team must have sufficient knowledge of advances in the science of resuscitation and prognostic factors associated with neurological outcomes in the elderly.

Blood ammonia, procalcitonin, heparin-binding protein, and brain natriuretic peptide are considered potential predictors for ROSC in patients with OHCA. (2326) However, we did not measure these values routinely in patients with cardiac arrest, and we are therefore unable to comment on their usefulness. Furthermore, although postresuscitation care, including therapeutic hypothermia, is well known to affect the outcomes of comatose patients, we did not determine significant differences in the number of patients with GCS $\leq 8$ undergoing therapeutic hypothermia. (27) As a result, we did not consider these variables for inclusion in the logistic regression model. Further studies may need to address these limitations.

This study has additional limitations. The first and most notable is the small study cohort. It is well known that the overall survival rate is very low in elderly populations, therefore resulting in lower OH-ROSC rate. (1) Second, accurate determination of the electrical rhythms of victims with ROSC prior to the arrival of the paramedics at the scene is difficult. Third, we only obtained clinical data for the patient's arrival at the ED and did not obtain records for the postresuscitation care in the ICU. Serum biomarkers such as S-10o, neuron-specific enolase, or evoked N2O response seem reliable predictors of neurological outcomes. $(28,29)$ However, these values are most accurate over $24 \mathrm{~h}$ after resuscitation and are thus unsuitable for the emergency setting. Fourth, we limited the study endpoint to neurological outcomes 3 months after OHCA. Long-term follow-up of the survivors was not conducted. Finally, our study sample was a small younger population, which had insufficient statistically power compared with the elderly populations. 


\section{Conclusion}

Lactate levels and GCS motor response measured immediately at ED arrival are likely to be useful to assess the neurological outcomes among elderly patients with OH-ROSC. Larger studies are essential to evaluate the prognostic factors that can predict neurological outcomes and to determine reliable cut-off parameters for elderly populations with OH-ROSC following sudden cardiac arrest.

\section{Acknowledgment}

The authors would like to thank Enago (www.enago.jp) for the English language review.

Figure 1. Optimal receiver operating characteristic curves for favorable neurological outcomes.

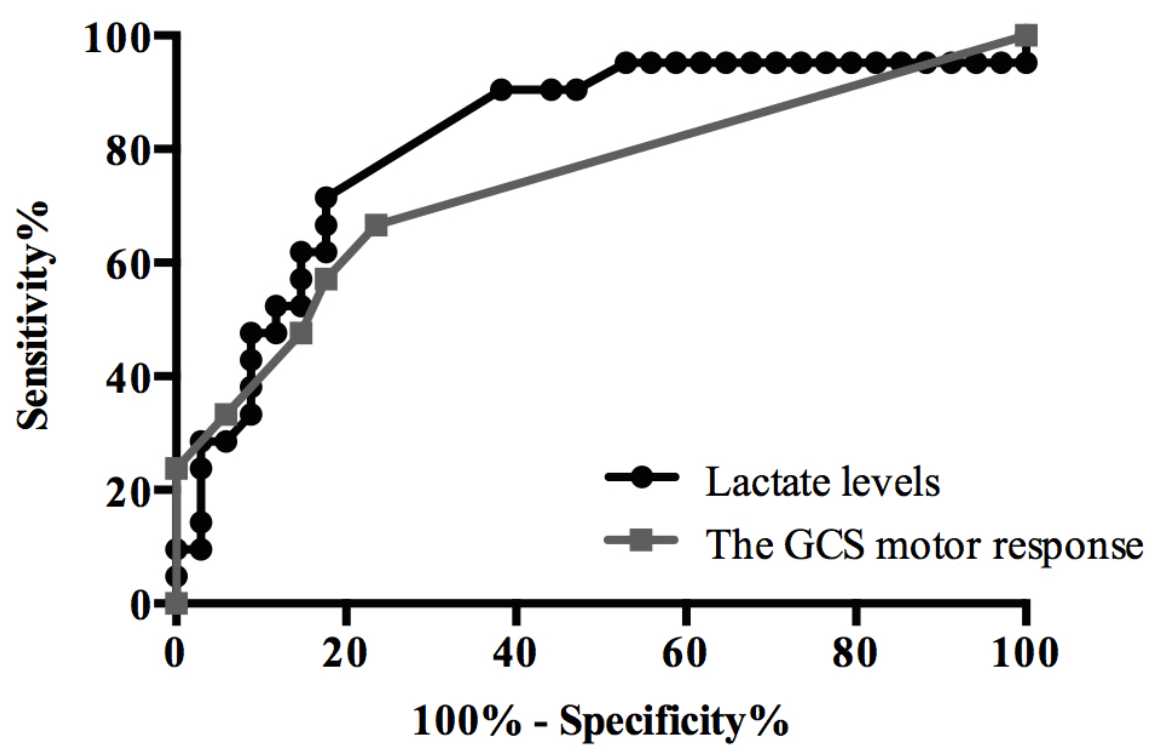

Areas under the receiver operating characteristic curves were $0.82[95 \%$ confidence interval $(\mathrm{CI})=0.70-0.94, \mathrm{p}<0.01]$ for lactate levels and $0.74(95 \% \mathrm{CI}=$ $0.60-0.88, \mathrm{p}<0.01)$ for the motor response on the Glasgow Coma Scale (GCS).

Table 1. Clinical characteristics and comparison between patients with favorable and unfavorable outcomes. 
SIGNA VITAE 2015; 10(1): 53 - 63

\begin{tabular}{|c|c|c|}
\hline Clinical variables & Favorable outcome $(n=21)$ & Unfavorable outcome $(\mathrm{n}=34)$ \\
\hline Age (years) -median (IQR) & $74(70-79)$ & $78(72-85)$ \\
\hline Female, $n(\%)$ & $3(14)$ & $13(38)$ \\
\hline Witnessed cardiac arrest, n (\%) & $18(86)$ & $25(74)$ \\
\hline Bystander CPR, $n(\%)$ & $14(67)$ & $18(53)$ \\
\hline \multicolumn{3}{|l|}{ First recorded fhythm on arriva1, $\mathrm{n}(\%)$} \\
\hline Asystole Pulse lass electrical activity/uniknown & $4(19)^{+}$ & $24(71)$ \\
\hline VF & $15(71)^{+}$ & $10(29)$ \\
\hline Pulpable carotid artery & $2(10)$ & 0 \\
\hline Defibrillation during ovt-of-hosp ital management, $\mathrm{n}$ (\%) & $15(71)^{+}$ & $12(35)$ \\
\hline \multicolumn{3}{|l|}{ Resugcitation duration (min) -median (IQR) } \\
\hline From collapse to notification to the EMS & $2(1-5)$ & $3(1-5)$ \\
\hline From starting BLS to achieving ROSC & $11(7-12)^{+}$ & $19(11-28)$ \\
\hline From achieving ROSC to arrival at the $\mathrm{ED}$ & $26(16-29)^{+}$ & $15(5-23)$ \\
\hline \multicolumn{3}{|l|}{ Cause of cardiac arrest, $\mathrm{n}(\%)$} \\
\hline Acute coron ary syndrome & $11(52)$ & $11(32)$ \\
\hline Other cardiac deiseases & $3(14)$ & $3(9)$ \\
\hline Choke & $3(14)$ & $8(24)$ \\
\hline Others & $4(19)$ & $12(35)$ \\
\hline $\operatorname{GCS} \leq 8, n(\%)$ & $14(67)^{+}$ & $33(97)$ \\
\hline Motor response of GCS score -median (IQR) & $3(1-5)^{+}$ & $1(1-1)$ \\
\hline Systolic blood pressure (mmHg) -median (IQR) & $87(71-105)$ & $95(68-113)$ \\
\hline Heart rate (beats/min) -median (IQR) & $100(86-115)$ & $97(82-114)$ \\
\hline Respiratory rate (beats/min) -median (IQR) & $12(6-20)$ & $12(6-19)$ \\
\hline Tympanic temperature $\left({ }^{\circ} \mathrm{C}\right)$-median $(\mathrm{IQR})$ & $35.8(35.5-36.2)$ & $359(35.1-36.6)$ \\
\hline $\mathrm{pH}-\operatorname{median}(\mathrm{IQR})$ & $7.33(7.30-7.37)^{+}$ & $7.10(6.94-7.28)$ \\
\hline alveolar-arterial oxygen difference - median $(I Q R)$ & $150(60-245)$ & $168(116-326)$ \\
\hline Base excess (mmolL)-median (IQR) & $-6.1(-9.1--4.1)^{\dagger}$ & $-13.7(-16.7--6.8)$ \\
\hline White blood cell $(j \mathrm{~L})$-median $(\mathrm{IQR})$ & $9895(6793-11925)$ & $9820(7960-12300)$ \\
\hline Hemoglobin ( $(\mathrm{g} / \mathrm{dL})$-madian (IQR) & $13.5(12.3-14.2)$ & $11.9(10.7-13.2)$ \\
\hline Hematocrit (\%)-median (IQR) & $40(37-43)$ & $35(33-40)$ \\
\hline Sodium (mEqL) -median (IQR) & $137(136-140)$ & $138(135-140)$ \\
\hline Pottasium $(\mathrm{mE} q \mathrm{~L})$ - $\operatorname{median}(\mathrm{IQR})$ & $3.8(3.6-4.4)$ & $4.2(3.7-5.1)$ \\
\hline Glucose (mg/dL) -median (IQR) & $207(157-281)$ & $247(178-277)$ \\
\hline Lactate (mgdL) -madian (IQR) & $51(31-69)^{+}$ & $74(69-96)$ \\
\hline Prothrombin intenational normalized ratio -madian ( $(\mathrm{IQR})$ & $1.1(1.1-1.2)$ & $1.3(1.1-1.6)$ \\
\hline D-dimer $(\mu \mathrm{g} / \mathrm{dL})$-madian $(\mathrm{IQR})$ & $3.4(1.5-5.0)$ & $8.5(4.5-22.8)$ \\
\hline Blood urea nitrogen (mg/dL) -median (IQR) & $17(14-20)$ & $23(18-27)$ \\
\hline Creatinine (mg/dL) -median (IQR) & $1.1(0.9-1.2)$ & $1.0(0.7-1.3)$ \\
\hline Albumin (g/dL)-median (IQR) & $3.4(3.1-3.6)$ & $3.5(3.0-3.9)$ \\
\hline Crea tinine phospho-kinase (IU L) -median (IQR) & $115(77-187)$ & $102(78-157)$ \\
\hline Creatine kinase $\mathrm{MB}(\mathrm{IU} / \mathrm{L})$-median $(\mathrm{IQR})$ & $2.0(1.3-2.7)$ & $2.1(1.5-3.0)$ \\
\hline APACHE III & $101(80-107)$ & $94(71-117)$ \\
\hline Therap evtic hypothermia, $\mathrm{n}(\%)$ & $13(62)$ & $12(35)$ \\
\hline
\end{tabular}

Table 2. Sensitivity and specificity of initial lactate cut-off points to predict a favorable neurological outcome using the respective receiver operating characteristic curve. 


\begin{tabular}{|c|c|c|c|c|c|}
\hline Lactateleveds (mg/dL) & Sensitivity & $95 \% \mathrm{CI}$ & Specificity & $95 \% \mathrm{CI}$ & Lilkelihood ratio \\
\hline$<85$ & 95 & $12-30.4$ & 100 & $89.7-100$ & - \\
\hline$<33.0$ & 28.6 & $113-522$ & 94.1 & $803-993$ & 49 \\
\hline$<50.0$ & 47.6 & $257-702$ & 882 & $723-9.7$ & 4.0 \\
\hline$<525$ & 524 & $29.8-743$ & 853 & $68.9-95.1$ & 3.6 \\
\hline$<61.0$ & 619 & $38.4-819$ & 82.4 & $655-932$ & 35 \\
\hline$<693$ & 905 & $69.6-99.8$ & 61.8 & $43.6-77.8$ & 2.4 \\
\hline$<705$ & 905 & $69.6-98.8$ & 559 & $379-72.8$ & 2.1 \\
\hline$<785$ & 952 & $762-999$ & 44.1 & $272-62.1$ & 1.7 \\
\hline
\end{tabular}

\section{References}

1. Akahane M, Tanabe S, Koike S, Ogawa T, Horiguchi H, Yasunaga H, et al. Elderly out-of-hospital cardiac arrest has worse outcomes with a family bystander than a non-family bystander. Int J Emerg Med 2012;5:41.

2. Johnson NJ, Salhi RA, Abella BS, Neumar RW, Gaieski DF, Carr BG. Emergency department factors associated with survival after sudden cardiac arrest. Resuscitation 2013;84:292-97.

3. Hayakawa K, Tasaki O, Hamasaki T, Sakai T, Shiozaki T, Nakagawa Y, et al. Prognostic indicators and outcome prediction model for patients with return of spontaneous circulation from cardiopulmonary arrest: the Utstein Osaka Project. Resuscitation 2011;82:874-80.

4. Wampler DA, Collett L, Manifold CA, Velasquez C, McMullan JT. Cardiac arrest is rare without prehospital return of spontaneous circulation. Prehosp Emerg Care 2012;16:451-5.

5. Nolan JP, Deakin CD, Soar J, Böttiger BW, Smith G. European Resuscitation Council. European resuscitation council guidelines for resuscitation 2005. Section 4. Adult advanced life support. Resuscitation 2005;67:S39-86.

6. Azlan A, Nidzwani S. Factors predicting outcome of cardiopulmonary resuscitation among elderly Malaysians: a retrospective study. Med J Malaysia 2012;67:278-83.

7. Sladjana A. A prediction survival model for out-of-hospital cardiopulmonary resuscitations. J Crit Care 2011;26:e11-8.

8. Mosier J, Itty A, Sanders A, Mohler J, Wendel C, Poulsen J, et al. Cardiocerebral resuscitation is associated with improved survival and neurological outcome from 
out-of-hospital cardiac arrest in elders. Acad Emerg Med 2010;17:269-75.

9. Cocchi MN, Miller J, Hunziker S, Carney E, Salciccioli J, Farris S, et al. The association of lactate and vasopressor need for mortality prediction in survivors of cardiac arrest. Minerva Anesthesiol 2011;77:1-9.

10. Kim J, Kim K, Lee JH, Jo YH, Kim T, RheeJE, et al. Prognostic implication of initial coagulopathy in out-of-hospital cardiac arrest. Resuscitation 2013;84:48-53.

11. Hasper D, von Haehling S, Storm C, Jörres A, Schefold JC. Changes in serum creatinine in the first 24 hours after cardiac arrest indicate prognosis: an observational cohort study. Crit Care 2009;13:R168.

12. Schefold JC, Storm C, Krüger A, Ploner CJ, Hasper D. The Glasgow Coma Scale is a predictor of good outcome in cardiac arrest patients treated with therapeutic hypothermia. Resuscitation 2009;80:658-61.

13. Ristagno G, Gullo A, Berlot G, Lucangelo U, Geheb E, Bisera J. Predictions of successful defibrillation in human victims of out-of-hospital cardiac arrest: a retrospective electrocardiographic analysis. Anaesth Intensive Care 20o8;36:4650.

14. Su YY, Yang QL, Pang Y, Lv XP. Evaluation of coma patients after cardiopulmonary resuscitation. Chin Med J 2005;118:1808-11.

15. Takase A, Sakamoto T, Okada Y. Arterial base excess after CPR: the relationship to CPR duration and characteristics related to outcome. Resuscitation 2007;73:394-9.

16. Levy DE, Caronna.JJ, Singer BH, Lapinski RH, Frydman H, Plum F. Predicting outcome from hypoxic-ischemic coma. JAMA 1985;253:1420-6.

17. Ajam K, Gold LS, Beck SS, Damon S, Phelps R, Rea TD. Reliability of the cerebral performance category to classify neurological status among survivors of ventricular fibrillation arrest: a cohort study. Scand J Trauma Resusc Emerg Med 2011;19:38.

18. Shapiro NI, Howell MD, Talmor D, Nathanson LA, Lisbon A, Wolfe RE, et al. Serum lactate as a predictor of mortality in emergency department patients with infection. Ann Emerg Med 2005;45:524-8.

19. Odom SR, Howell MD, Silva GS, Nielsen VM, Gupta A, Shapiro NI, et al. Lactate clearance as a predictor of mortality in trauma patients. J Trauma Acute Care Surg 2013;74:999-1004.

20. del Portal DA, Shofer F, Mikkelsen ME, Dorsey PJJr, Gaieski DF, Goyal M, et al. Emergency department lactate is associated with mortality in older adults admitted with and without infections. Acad Emerg Med 2010;17:260-8.

21. Shinozaki K, Oda S, Sadahiro T, Nakamura M, Hirayama Y, Watanabe E, et al. Blood ammonia and lactate levels on hospital arrival as a predictive biomarker in 
patients with out-of-hospital cardiac arrest. Resuscitation 2011;82:404-9.

22. Bonnin MJ, Pepe PE, Clark PS Jr. Survival in the elderly after out-of-hospital cardiac arrest. Crit Care Med 1993;21:1645-51.

23. Scott PA, Barry J, Roberts PR, Morgan JM. Brain natriuretic peptide for the prediction of sudden cardiac death and ventricular arrhythmias: a meta-analysis. Eur J Heart Fail 2009;11:958-66.

24. Lin CH, Chi CH, Wu SY, Hsu HC, Chang YH, Huang YY, et al. Prognostic values of blood ammonia and partial pressure of ammonia on hospital arrival in out-ofhospital cardiac arrests. AmJ Emerg Med 2013;31:8-15.

25. Annborn M, Dankiewicz J, Erlinge D, Hertel S, Rundgren M, Smith JG, et al. Procalcitonin after cardiac arrest - An indicator of severity of illness, ischemia reperfusion injury and outcome. Resuscitation 2013;84:782-7.

26. Dankiewicz J, Linder A, Annborn M, Rundgren M, Friberg H. Heparin-binding protein: An early indicator of critical illness and predictor of outcome in cardiac arrest. Resuscitation 2013;84:935-9.

27. Marko N. Hypothermia during percutaneous coronary intervention in comatose survivors of cardiac care. SIGNA VITAE. 2010;5:13-6.

28. Einav S, Kaufman N, Algur N, Kark JD. Modeling serum biomarker S1oo beta and neuron-specific enolase as predictors of outcome after out-of-hospital cardiac arrest: an aid to clinical decision making.J Am Coll Cardiol 2012;60:304-11.

29. Rothstein TL. Therapeutic hypothermia and reliability of somatosensory evoked potentials in predicting outcome after cardiopulmonary arrest. Neurocrit Care 2012;17:146-9.

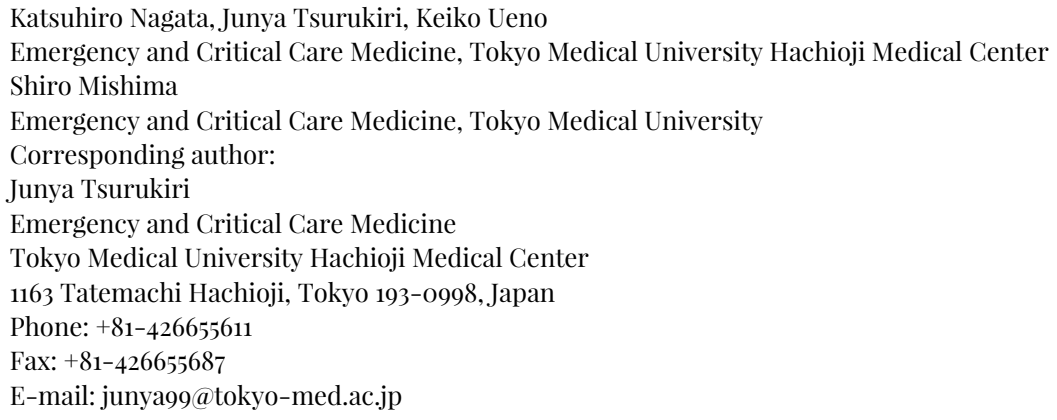

